\title{
Dipole-coupled emitters as deterministic entangled photon-pair sources
}

\author{
Derek S. Wang, ${ }^{*}$ Tomáš Neuman, ${ }^{*}$ and Prineha Narang $\odot^{\dagger}$ \\ Harvard John A. Paulson School of Engineering and Applied Sciences, Harvard University, Cambridge, Massachusetts 02138, USA
}

(Received 16 June 2020; revised 23 September 2020; accepted 6 November 2020; published 7 December 2020)

\begin{abstract}
Scalable quantum systems require deterministic entangled photon pair sources. Here, we demonstrate a scheme that uses a dipole-coupled emitter pair to deterministically emit polarization-entangled photon pairs. Based on this scheme, we predict spectroscopic signatures and quantify the entanglement with realistic material parameters. In addition, we describe how the Bell state fidelity and efficiency can be optimized by precisely tuning transition frequencies. Finally, we discuss how defect emitters are natural candidates for the proposed scheme, offering numerous advantages including flexible on-chip photonic integration and tunable emission properties via external fields, electromagnetic environments, and defect selection.
\end{abstract}

DOI: 10.1103/PhysRevResearch.2.043328

\section{INTRODUCTION}

Nonclassical states of light are important resources for quantum technologies, such as quantum information processing, networking, and metrology [1]. Entangled photon pairs, in particular, have applications in solid-state quantum repeaters, a crucial component of long-distance quantum networking that overcomes transmission loss by leveraging the effects of entanglement swapping and quantum teleportation [2-7]. Despite the diverse applications for such nonclassical states of light, methods for generating them deterministically remain limited. Currently, successful approaches are based on spontaneous parametric down-conversion [8-10] or spontaneous four-wave mixing $[11,12]$ with high performance [13-15]. A major drawback of such methods is that the number of photon pairs generated follows a Poissonian distribution [16], rendering the pair generation efficiency too low for scalable quantum systems [3]. Semiconductor quantum dots can deterministically emit entangled photon pairs via biexciton decay cascade [2,17-24] with high fidelity and emission efficiency. This mechanism, however, requires careful engineering of quantum dots and pumping schemes that poses a technological challenge, motivating the search for alternative pathways to the generation pairs.

In this article, we provide the theoretical basis for a deterministic entangled photon pair source from a pair of dipole-coupled [25] three-level quantum emitters. Each emitter consists of a ground state and two optically active electronic excited states with mutually orthogonal transition dipole moments. The emergent electronic structure of

\footnotetext{
*These two authors contributed equally.

†prineha@seas.harvard.edu
}

Published by the American Physical Society under the terms of the Creative Commons Attribution 4.0 International license. Further distribution of this work must maintain attribution to the author(s) and the published article's title, journal citation, and DOI. the composite system then allows for the implementation of a radiative decay cascade from a symmetric doubly excited state of the pair, which results in the emission of two entangled photons of orthogonal polarization. We analyze the resulting emission spectra to note qualitative signatures of entanglement, especially in the cross-correlation spectrum.

To better quantify the entanglement of the emitted photon pairs in continuous frequency space, we calculate their entanglement entropy $S$ and Bell state fidelity $\mathcal{F}$. Importantly, Bell state fidelity $\mathcal{F}$ can be optimized at the expense of the entanglement entropy $S$ by tuning the defect transition dipole moments and concentrating the probability density within the states of interest, or the ideal Bell state. In addition, we find that the entanglement measures of the emitted photons are robust to relative differences in frequency between the intermediate states, while the fidelity in the presence of phonon-based dephasing is limited when the dephasing rate is on the order of the radiative rate or higher. We also present a method of initializing the system with orthogonally polarized continuous wave lasers that involves two-photon absorption to enable Rabi oscillations between the ground and symmetric doubly excited state of the pair.

This scheme has the advantage of requiring only emitters with well understood singly-excited states that can be realized by a variety of physical systems, whereas accurate determination of the energetics of multiply excited states from first principles remains a challenge. We specifically discuss the applicability of defect emitters, given their fixed geometries enabling stable dipole coupling, diverse symmetries that allow nondegenerate transitions with orthogonal transition dipole moments, and emission properties that can be tailored via external fields. In addition, the chemical selection space of defect systems is vast. The present scheme is, however, likely amenable to dipole-coupled quantum dots or molecules as well, although they may lack certain advantages of dipolecoupled defect centers. The ability to generate entangled photon pairs from defects would enable on-chip integration [12] with quantum memories and emitters, minimizing the 


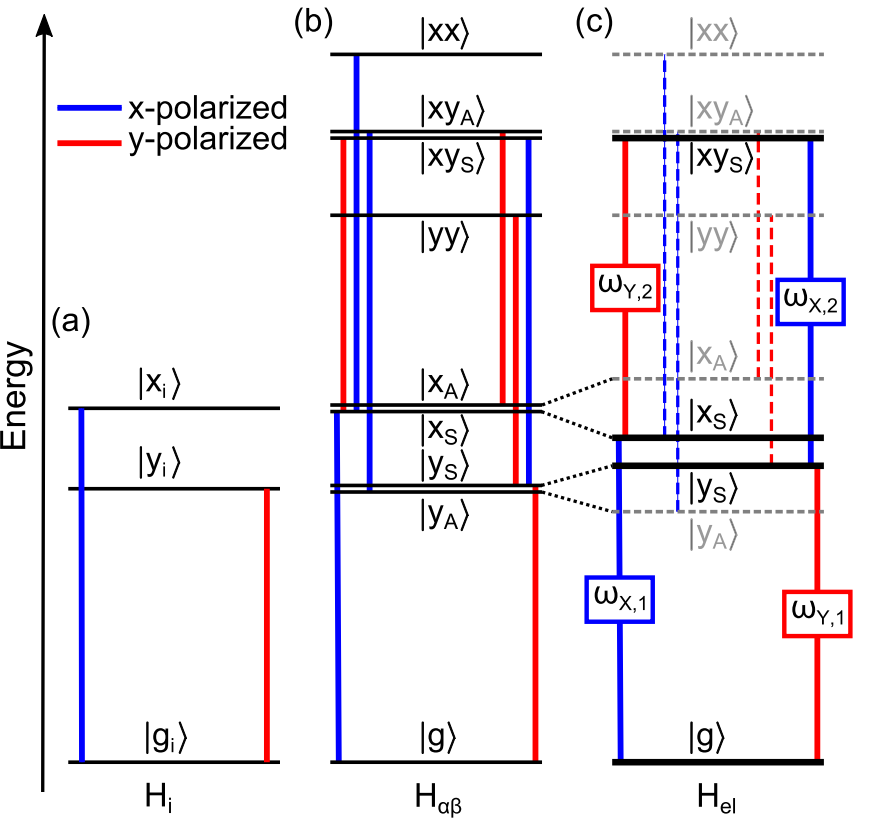

FIG. 1. Energy level diagrams and dipole-allowed transitions, where allowed $x$ - and $y$-polarized transitions are in red and blue, respectively. (a) A single three-level emitter. (b) Two distantly separated three-level emitters such that dipole coupling is negligible. (c) Two dipole-coupled, three-level emitters. Bolded states and transitions (with transition frequencies $\omega_{X, 1}, \omega_{X, 2}, \omega_{Y, 1}$, and $\omega_{Y, 2}$ ) are accessible when the system is prepared in $\left|x y_{\mathrm{S}}\right\rangle$.

need to transduce photons from source to storage to emission in quantum technologies.

\section{MODEL}

The system consists of two three-level systems denoted by $i \in\{\alpha, \beta\}$. Each three-level system consists of a ground state $\left|g_{i}\right\rangle$, an excited state $\left|x_{i}\right\rangle$ with energy $\hbar \omega_{x}$ and transition dipole moment $\boldsymbol{d}_{x_{i}}=\left\langle x_{i}|\mathrm{e} \boldsymbol{r}| g_{i}\right\rangle=d_{x_{i}} \hat{x}$, and an excited state $\left|y_{i}\right\rangle$ with energy $\hbar \omega_{y}$ and transition dipole moment $\boldsymbol{d}_{y_{i}}=$ $\left\langle y_{i}|\mathrm{e} \boldsymbol{r}| g_{i}\right\rangle=d_{y_{i}} \hat{y}$, where $\boldsymbol{r}$ is the position operator and $\mathrm{e}$ is the electron charge. The energy level diagram and dipoleallowed transitions are plotted in Fig. 1(a). The Hamiltonian $H_{i}$ of each isolated three-level system can be written as $H_{i}=$ $\hbar \omega_{x}\left|x_{i}\right\rangle\left\langle x_{i}\left|+\hbar \omega_{y}\right| y_{i}\right\rangle\left\langle y_{i}\right|$.

When emitters $\alpha$ and $\beta$ at positions $\boldsymbol{r}_{\alpha}$ and $\boldsymbol{r}_{\beta}$, respectively, are brought close and couple via electric dipole interactions, the total electronic Hamiltonian $H_{\mathrm{el}}$ can be written in the product space of the two three-level systems as

$$
H_{\mathrm{el}}=H_{\alpha \beta}+H_{\mathrm{dip}},
$$

where $H_{\alpha \beta}=H_{\alpha}+H_{\beta}$, and the dipole-coupling Hamiltonian $H_{\text {dip }}$, in the rotating wave approximation (RWA) where we have dropped double (de)excitations, is given by

$$
H_{\text {dip }}=\sum_{p q \in\{x, y\}} J_{p q}(|g p\rangle\langle q g|+| q g\rangle\langle g p|),
$$

where $|r s\rangle \equiv\left|r_{\alpha}\right\rangle\left|s_{\beta}\right\rangle$ with $r, s \in\{g, x, y\}$, and transition dipole moments are real. Although we assume the emitter states do not have permanent dipole moments, we can
TABLE I. Eigenstates and eigenenergies of $H_{\mathrm{el}}$.

\begin{tabular}{ccc}
\hline \hline & Eigenstate & Eigenenergy \\
\hline 1 & $|g\rangle \equiv|g g\rangle$ & $\hbar \omega_{g}=0$ \\
2 & $\left|y_{\mathrm{A}}\right\rangle \equiv \frac{1}{\sqrt{2}}(|g y\rangle-|y g\rangle)$ & $\hbar \omega_{y_{\mathrm{A}}}=\hbar \omega_{y}-J_{y y}$ \\
3 & $\left|y_{\mathrm{S}}\right\rangle \equiv \frac{1}{\sqrt{2}}(|g y\rangle+|y g\rangle)$ & $\hbar \omega_{y_{\mathrm{S}}}=\hbar \omega_{y}+J_{y y}$ \\
4 & $\left|x_{\mathrm{S}}\right\rangle \equiv \frac{1}{\sqrt{2}}(|g x\rangle+|x g\rangle)$ & $\hbar \omega_{x_{\mathrm{S}}}=\hbar \omega_{x}-J_{x x}$ \\
5 & $\left|x_{\mathrm{A}}\right\rangle \equiv \frac{1}{\sqrt{2}}(|g x\rangle-|x g\rangle$ & $\hbar \omega_{x_{\mathrm{A}}}=\hbar \omega_{x}+J_{x x}$ \\
6 & $|y y\rangle$ & $\hbar \omega_{y y}=2 \hbar \omega_{y}$ \\
7 & $\left|x y_{\mathrm{S}}\right\rangle \equiv \frac{1}{\sqrt{2}}(|x y\rangle+|y x\rangle)$ & $\hbar \omega_{x y_{\mathrm{S}}}=\hbar\left(\omega_{x}+\omega_{y}\right)$ \\
8 & $\left|x y_{\mathrm{A}}\right\rangle \equiv \frac{1}{\sqrt{2}}(|x y\rangle-|y x\rangle)$ & $\hbar \omega_{x y_{\mathrm{A}}}=\hbar \omega_{x y_{\mathrm{S}}}$ \\
9 & $|x x\rangle$ & $\hbar \omega_{x x}=2 \hbar \omega_{x}$ \\
\hline \hline
\end{tabular}

include the interactions of static dipoles as diagonal terms in the single-emitter subspace. We also assume the orbitals of neighboring emitters do not hybridize in the interdefect ranges considered to be a few to tens of nanometers because, for defects specifically, orbitals can be localized within a few angstroms [26-30]. The dipole interaction energy $J_{p q}$ is [25]

$$
J_{p q}=\frac{\left|\boldsymbol{d}_{p_{\alpha}}\right| \boldsymbol{d}_{q_{\beta}} \mid}{4 \pi \epsilon_{0} \epsilon_{r}\left|\boldsymbol{r}_{\alpha}-\boldsymbol{r}_{\beta}\right|^{3}}\left[\boldsymbol{e}_{p_{\alpha}} \cdot \boldsymbol{e}_{q_{\beta}}-3\left(\boldsymbol{e}_{p_{\alpha}} \cdot \boldsymbol{n}\right)\left(\boldsymbol{e}_{q_{\beta}} \cdot \boldsymbol{n}\right)\right],
$$

where $\epsilon_{r}$ is the relative permittivity of the host material, $\boldsymbol{e}_{s_{i}}$ is the unit vector of the dipole moment $\boldsymbol{d}_{s_{i}}$, and $\boldsymbol{n}$ is the unit vector of $\boldsymbol{r}_{\alpha}-\boldsymbol{r}_{\beta}$. The coupling rates $J_{p q}$ can be calculated from the $a b$ initio transition charge densities of the respective electronic transitions or can be obtained directly from the $a b$ initio calculations of the excited states of the coupled emitter pair. Since transition dipole moments can be on the order of $\sim 1$ eA in small- to medium-sized molecules [31] on the same size scale as defect emitters, we estimate that emitters spaced a few $\mathrm{nm}$ apart can have dipole interaction energies on the order of tens of $\mu \mathrm{eV}$.

Assuming for the sake of simplicity that $\boldsymbol{n}$ lies on the $x$ axis, and that the dipole moments of the same polarizations of emitters $\alpha$ and $\beta$ are identical $\left(d_{x} \equiv d_{x_{\alpha}}=d_{x_{\beta}}\right.$ and $d_{y} \equiv$ $\left.d_{y_{\alpha}}=d_{y_{\beta}}\right), H_{\mathrm{el}}$ can be diagonalized to produce nine eigenstates with eigenenergies listed in Table I. The subscripts " $A$ " and "S" stand for "antisymmetric" and "symmetric" combinations, respectively. The energy diagram of the eigenstates of $H_{\alpha \beta}$ and $H_{\mathrm{el}}$ and their dipole-allowed transitions, derived from the dipole operator $\boldsymbol{d}$ listed in Table II in Appendix A, are plotted in Figs. 1(b) and 1(c). Notably, direct transitions between symmetric and antisymmetric states are dipole forbidden. From the energy diagram corresponding to $H_{\mathrm{el}}$, we see that a polarization-entangled photon pair can be emitted when the system is prepared in $\left|x y_{S}\right\rangle$ and irreversibly decays.

We calculate emission spectra into free space by coupling the emitter system initially prepared in $\left|x y_{\mathrm{S}}\right\rangle$ to an unexcited continuum of photon modes and solving the time-dependent Schrödinger equation under the Weisskopf-Wigner approximation [32], similar to the approach introduced in Ref. [33]. The total Hamiltonian $H$ of the coupled emitter-photon system is

$$
H=H_{\mathrm{el}}+H_{\mathrm{ph}}+H_{\mathrm{el}-\mathrm{ph}} .
$$


(a)

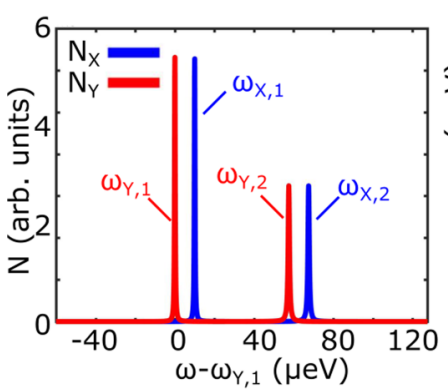

(b)

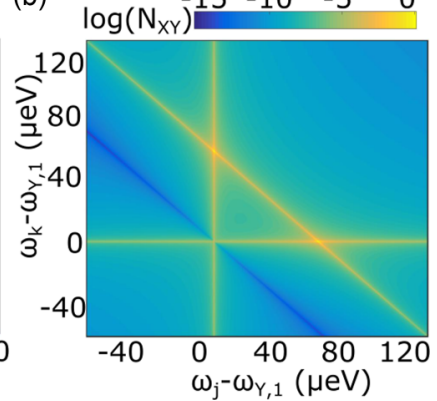

FIG. 2. Spectra of emitted polarization-entangled photon pair. (a) The single-photon spectra $N_{X}\left(\omega_{j}\right)$ and $N_{Y}\left(\omega_{k}\right)$ corresponding to $x$ - and $y$-polarized photons, respectively, and (b) the crosscorrelation function $N_{X Y}\left(\omega_{j}, \omega_{k}\right)$. Based on experimentally observed ranges of parameters, we set $\omega_{y_{\mathrm{S}}}=2 \mathrm{eV}, \omega_{x_{\mathrm{S}}}=\omega_{y_{\mathrm{S}}}+10 \mu \mathrm{eV}, d_{x}=$ $d_{y}=1 \mathrm{e} \AA,\left|\boldsymbol{r}_{\alpha}-\boldsymbol{r}_{\beta}\right|=5 \mathrm{~nm}, \epsilon_{r}=2$, and $\gamma_{g, y_{\mathrm{S}}}=0.2 \mu \mathrm{eV}$.

The photonic Hamiltonian $H_{\mathrm{ph}}$ is $H_{\mathrm{ph}}=\sum_{j l} \hbar \omega_{j} a_{j l}^{\dagger} a_{j l}$, where $a_{j l}\left(a_{j l}^{\dagger}\right)$ are annihilation (creation) operators of the $j$ th mode in the electromagnetic vacuum of free space with polarization $l \in\{X, Y\}$ and energy $\hbar \omega_{j}$. In $H_{\mathrm{ph}}$, we have dropped the zero-point contribution with no loss of generality. The electron-photon coupling Hamiltonian in the RWA and dipole approximation is $H_{\mathrm{el}-\mathrm{ph}}=-\sum_{o p j l} \mathcal{E}_{j l} \cdot \boldsymbol{d}_{o p}|o\rangle\langle p| a_{j l}^{\dagger}+$ H.c., where $\mathcal{E}_{j l}$ is the electric field with magnitude $\mathcal{E}$ in the $l$ direction that we assume to be constant for all $j$, and $\boldsymbol{d}_{o p}=\langle o|\mathrm{e}| p\rangle$ with $|o\rangle$ and $|p\rangle$ being quantum states of the combined twoemitter system.

The ansatz for a general electron-photon wave function, noting that for a system prepared in $\left|x y_{\mathrm{S}}\right\rangle$ there can be a maximum of two excitations distributed among the electronic and photonic states, is

$$
\begin{aligned}
|\Psi(t)\rangle= & \sum_{j k} c_{j k}^{g}|g\rangle a_{j X}^{\dagger} a_{k Y}^{\dagger}|\mathrm{vac}\rangle+\sum_{j} c_{j}^{x_{\mathrm{S}}}\left|x_{\mathrm{S}}\right\rangle a_{j Y}^{\dagger}|\mathrm{vac}\rangle \\
& +\sum_{j} c_{j}^{y_{\mathrm{S}}}\left|y_{\mathrm{S}}\right\rangle a_{j X}^{\dagger}|\mathrm{vac}\rangle+c^{x y_{\mathrm{S}}}\left|x y_{\mathrm{S}}\right\rangle|\mathrm{vac}\rangle
\end{aligned}
$$

where $j$ and $k$ are indices for the continuum of photon modes and $|\mathrm{vac}\rangle$ is the photon vacuum state, and $c_{j k}^{g}, c_{j}^{x_{\mathrm{S}}}, c_{j}^{y_{\mathrm{S}}}$, and $c^{x y_{\mathrm{s}}}$ are time-dependent amplitudes. We have dropped all antisymmetric, $|y y\rangle$, and $|x x\rangle$ terms because the emitter system is initially prepared in $\left|x y_{\mathrm{S}}\right\rangle$.

We solve the time-dependent Schrödinger equation under the Weisskopf-Wigner approximation to find the final state of the electron-photon system under irreversible spontaneous decay [33]:

$$
|\Psi(\infty)\rangle=\sum_{j k} c_{j k}^{g}(\infty)|g\rangle a_{j X}^{\dagger} a_{k Y}^{\dagger}|\mathrm{vac}\rangle,
$$

where

$$
c_{j k}^{g}(\infty)=\frac{\frac{-\Omega_{g, x_{\mathrm{S}}} \Omega_{x_{\mathrm{S}}, x_{\mathrm{S}}}}{i \omega_{x_{\mathrm{S}}}-i \omega_{j}+\gamma_{g, x_{\mathrm{S}}}}+\frac{-\Omega_{g, y_{\mathrm{S}}} \Omega_{y_{\mathrm{S}}, x_{\mathrm{S}}}}{i \omega_{y_{\mathrm{S}}}-i \omega_{k}+\gamma_{g, y_{\mathrm{S}}}}}{i\left(\omega_{x y_{\mathrm{S}}}-\omega_{j}-\omega_{k}\right)+\gamma_{x_{\mathrm{S}}, x y_{\mathrm{S}}}+\gamma_{y_{\mathrm{S}}, x y_{\mathrm{S}}}},
$$

$\Omega_{o p}=-\mathcal{E}\left|\boldsymbol{d}_{o p}\right| / \hbar, \quad \gamma_{o p}=\mathcal{E}^{2}\left|\boldsymbol{d}_{o p}\right|^{2} / \Delta$, and $\Delta$ is the frequency spacing. Further details on obtaining Eq. (7) are in Appendix B.

\section{ENTANGLED PHOTON PAIRS}

We explore the physical parameters that result in photon pair entanglement. First, we calculate spectra for a photon pair emitted by a dipole-coupled emitter pair and note spectral signatures of entanglement. We optimize the Bell state fidelity by tuning transition frequencies. These changes can be implemented by appropriate selection of an emitter system or applying external fields.

The emission cascade caused by the radiative decay of the optically excitable $\left|x y_{\mathrm{S}}\right\rangle$ state of the composite emitter-emitter system results in the emission of $x$ - and $y$-polarized photons whose number spectra are generally distinct, as we show in Fig. 2(a) for the parameters given in the figure caption. We calculate the number spectra, or the probability of finding an $x$-polarized (y-polarized) photon with frequency $\omega_{j}\left[\omega_{k}\right]$, as $N_{X}\left(\omega_{j}\right)=\sum_{k}\left|c_{j k}^{\mathrm{g}}\right|^{2}\left[N_{Y}\left(\omega_{k}\right)=\sum_{j}\left|c_{j k}^{\mathrm{g}}\right|^{2}\right]$. While the $x$ polarized photon spectrum $N_{X}\left(\omega_{j}\right)$ (blue curve) peaks around the frequencies $\omega_{X, 1}$ and $\omega_{X, 2}$, the maxima of the $y$-polarized spectrum are found at $\omega_{Y, 1}$ and $\omega_{Y, 2}$, corresponding to the respective transitions in the two-photon cascade depicted in Fig. 1(c) as blue and red lines.

The emitted $x$ - and $y$-polarized photons of different frequencies exhibit nontrivial correlations. We plot in Fig. 2(a) the cross-correlation function $N_{X Y}\left(\omega_{j}, \omega_{k}\right)=\left|c_{j k}^{g}\right|^{2}$, measuring the probability to simultaneously detect an $x$-polarized photon of frequency $\omega_{j}$ and a $y$-polarized photon of frequency $\omega_{k}$. The cross-correlation function features local maxima at two points. When an $x$-polarized photon is detected with frequency $\omega_{X, 1}$, the $y$-polarized photon is most likely detected with frequency $\omega_{Y, 2}$ [i.e., $N_{X Y}\left(\omega_{X, 1}, \omega_{Y, 2}\right)$ is a maximum], and when an $x$-polarized photon is detected with frequency $\omega_{X, 2}$, the probability of simultaneously finding a $y$-polarized photon peaks for frequency $\omega_{Y, 1}$. This correlated behavior for a pure state is an intuitive signature of bipartite entanglement.

We consider two metrics to rigorously quantify the entanglement of emitted photon pairs. The first metric is the entanglement entropy $S$ [34-36]:

$$
S=-\sum_{n}\left|\lambda_{n}\right|^{2} \log _{2}\left|\lambda_{n}\right|^{2}
$$

We find the singular values $\lambda_{n}$ by Schmidt decomposition of the photonic portion $\left|\Psi_{\mathrm{ph}}\right\rangle$ of the final state in Eq. (6):

$$
\left|\Psi_{\mathrm{ph}}\right\rangle=\sum_{n} \lambda_{n} b_{n X}^{\dagger} c_{n Y}^{\dagger}|\mathrm{vac}\rangle
$$

where the creation operators $b_{n X}^{\dagger}=\sum_{j} \psi_{n j} a_{j X}^{\dagger}$ and $c_{n Y}^{\dagger}=\sum_{k} \phi_{n k} a_{k Y}^{\dagger}$ in the Schmidt basis, $\lambda_{n}$ represent wave function coefficients in decreasing order with $n$ starting at 0 and incrementing by 1 , and $\psi_{n j}$ and $\phi_{n k}$ are the eigenfunctions of $c_{j k}^{g}$. The entanglement entropy is zero if the state is factorizable and greater than zero for an entangled state.

In protocols based on entanglement, it is often convenient to work directly with Bell states, so the second and third metrics we consider are the Bell state efficiency $\eta$ and fidelity $\mathcal{F}$, where the Bell state $\left|\Psi^{+}\right\rangle=\frac{1}{\sqrt{2}}(|10\rangle+|01\rangle)$ in the logical basis. To write $\left|\Psi_{\mathrm{ph}}\right\rangle$ in the logical basis, we assign the Schmidt states defined by the two pairs of $b_{n X}^{\dagger}$ and $c_{n Y}^{\dagger}$ with 
(a)

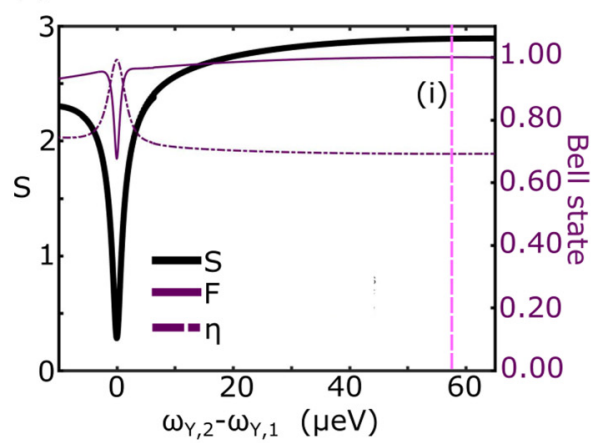

(b)

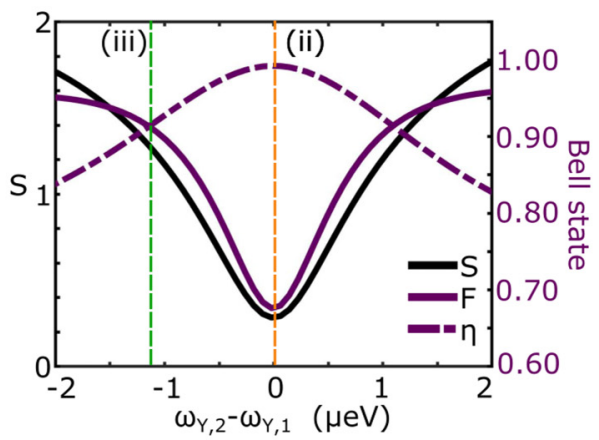

(c)

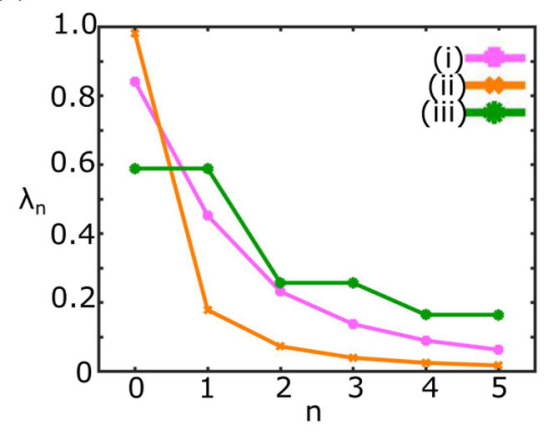

FIG. 3. Entanglement optimization. (a) Entanglement entropy $S$, Bell state efficiency $\eta$, and Bell state fidelity $\mathcal{F}$ for varying $\omega_{Y, 2}-\omega_{Y, 1}=$ $\omega_{X, 2}-\omega_{X, 1}$, effected by changing $d_{x}$. The pink line (i) corresponds to the conditions in Fig. 2. (b) Magnified near $\omega_{Y, 2}-\omega_{Y, 1}=0$. Both $S$ and $\mathcal{F}$ are minimized at (ii), and both $\eta$ and $\mathcal{F}>0.90$ at (iii). (c) Singular values (wave function coefficients) of entangled photon pairs corresponding to conditions marked by (i), (ii), and (iii) in Figs. 3(a) and 3(b).

the highest $\lambda_{n}$ to $|10\rangle$ and $|01\rangle$, respectively:

$$
\left|\Psi_{\mathrm{ph}}\right\rangle=\lambda_{0}|10\rangle+\lambda_{1}|01\rangle+\sum_{n \geqslant 2} \lambda_{n} b_{n X}^{\dagger} c_{n Y}^{\dagger}|\mathrm{vac}\rangle .
$$

We trace out all states where $n \geqslant 2$ to write the reduced density matrix $\rho_{\mathrm{R}}$ as

$$
\rho_{\mathrm{R}}=\left(\lambda_{0}^{2}+\lambda_{1}^{2}\right)|\psi\rangle\left\langle\psi\left|+\sum_{n \geqslant 2} \lambda_{n}^{2}\right| 00\right\rangle\langle 00|,
$$

where $|\psi\rangle=1 / \sqrt{\lambda_{0}^{2}+\lambda_{1}^{2}}\left(\lambda_{0}|10\rangle+\lambda_{1}|01\rangle\right)$. The efficiency $\eta$ of collecting $|10\rangle$ and $|01\rangle$ is

$$
\eta=\lambda_{0}^{2}+\lambda_{1}^{2},
$$

and the Bell state fidelity $\mathcal{F}=\left|\left\langle\Psi^{+} \mid \psi\right\rangle\right|^{2}$ is

$$
\mathcal{F}=\frac{1}{2} \frac{\left(\lambda_{0}+\lambda_{1}\right)^{2}}{\lambda_{0}^{2}+\lambda_{1}^{2}} \text {. }
$$

In Fig. 3 we show how the entanglement can be optimized by tuning emitter parameters. In Fig. 3(a), we sweep $d_{x}$ while holding all other physical parameters described in Fig. 2 constant. As a result, $\omega_{Y, 2}\left[\omega_{X, 2}\right]$ shifts relative to $\omega_{Y, 1}\left[\omega_{X, 1}\right]$, modulating the distance between peaks of the single-photon spectrum of a given polarization. Notably, for the exact conditions plotted in Fig. $2, d_{x}=d_{y}, \mathcal{F}$ is nearly 1 while $\eta=0.69$. In Fig. 3(b), we zoom into the region around $\omega_{Y, 2}=\omega_{Y, 1}$, corresponding to $d_{x}=\frac{1}{\sqrt{2}} d_{y}$. Here we observe a minimum in $S$ and $\mathcal{F}$ and a maximum in $\eta$. The entanglement entropy drops here because the frequency of a photon with a given polarization emitted by one of the two decay paths is the same as the photon with a given polarization emitted via the other decay path, so photon pairs emitted by either of the two decay paths are identical. The finite linewidth of the emissions, however, permits entanglement among photon modes within this peak, so the entanglement entropy does not bottom out at 0 .

$\mathcal{F}$ and $\eta$ of the Bell pair change in opposite directions surrounding the minimum of $\mathcal{F}$ and $S$. To understand the origin of this observation, in Fig. 3(c) we plot the first few Schmidt coefficients $\lambda_{n}$ when: (i) $d_{x}=d_{y}$, corresponding to the state analyzed in Fig. 2, (ii) $S$ and $\mathcal{F}$ are minimized, and (iii) both $\eta$ and $\mathcal{F}>0.90$. In (i), we see that $\lambda_{n}$ come in pairs, mean- ing that this state is a superposition of many high-fidelity, polarization-entangled Bell states. In (ii), where $S$ and $\mathcal{F}$ are minimized, $\lambda_{n}$ decays more quickly than in (i). Nearly all of the population is concentrated in the first state, so there are fewer entangled states, lowering $S$. A balance is achieved in (iii) where probability density is concentrated within the first two pairs of entangled states, but $\lambda_{0} \neq \lambda_{1}$. Thus, by tuning the transition frequencies, we can optimize for $\mathcal{F}$ or $\eta$. The entanglement measures are robust to changes in $\omega_{X, 1}-\omega_{Y, 1}$, and $\mathcal{F}$ and $\mathcal{S}$ are relatively unaffected by up to an order of magnitude increase in $\gamma_{g, y_{S}}$, as shown in Appendix C. We also show that the fidelity in the presence of dephasing is limited by the radiative linewidth $\sim \gamma_{g, y S}$ in Appendix D, suggesting optimal operation under dilution fridge conditions. Finally, we note that the emitted photon pairs can undergo entanglement distillation to further enhance the Bell state fidelity [34,37-40].

\section{PUMPING SCHEME}

We next describe a possible pumping scheme involving two-photon absorption via continuous wave lasers to initialize the composite emitter system in the doubly excited $\left|x y_{\mathrm{S}}\right\rangle$ state from which the entangled photon pair is emitted after radiative decay cascade, analogous to schemes proposed for the Mølmer-Sørensen gate [41] and biexcitonic semiconducting quantum dots [42]. We consider a general scenario where the transition frequencies $\omega_{\mathrm{X}, 1} \neq \omega_{\mathrm{X}, 2}$ and $\omega_{\mathrm{Y}, 1} \neq \omega_{\mathrm{Y}, 2}$. In this case each electronic transition of the system can be selectively addressed by choosing the right polarization and frequency of an external laser drive. In particular, the following two-photon driving Hamiltonian $H$ can be realized if two lasers of polarizations and amplitudes $\mathcal{E}_{x} \hat{x}$ and $\mathcal{E}_{y} \hat{y}$, and respective frequencies $\tilde{\omega}_{\mathrm{X}, 1}=\omega_{\mathrm{X}, 1}+\delta$ and $\tilde{\omega}_{\mathrm{Y}, 2}=\omega_{\mathrm{Y}, 2}-\delta$, are used to illuminate the system:

$$
\begin{aligned}
\frac{H_{\text {drive }}}{\hbar}= & |g\rangle\left\langle x_{\mathrm{S}}\right| \sqrt{2}\left(\mathcal{E}_{x} e^{-\mathrm{i} \tilde{\omega}_{\mathrm{X}, 1} t}+\mathcal{E}_{x}^{*} e^{\mathrm{i} \tilde{\omega}_{\mathrm{X}, 1} t}\right) \\
& +\left|x_{\mathrm{S}}\right\rangle\left\langle x y_{\mathrm{S}}\right|\left(\mathcal{E}_{y} e^{-\mathrm{i} \tilde{\omega}_{\mathrm{Y}, 2} t}+\mathcal{E}_{y}^{*} e^{\mathrm{i} \tilde{\omega}_{\mathrm{Y}, 2} t}\right) \\
& +|g\rangle\left\langle y_{\mathrm{S}}\right| \sqrt{2}\left(\mathcal{E}_{y} e^{-\mathrm{i} \tilde{\omega}_{\mathrm{Y}, 2} t}+\mathcal{E}_{y}^{*} e^{\mathrm{i} \tilde{\omega}_{\mathrm{Y}, 2} t}\right) \\
& +\left|y_{\mathrm{S}}\right\rangle\left\langle x y_{\mathrm{S}}\right|\left(\mathcal{E}_{x} e^{-\mathrm{i} \tilde{\omega}_{\mathrm{X}, 1} t}+\mathcal{E}_{x}^{*} e^{\mathrm{i} \tilde{\omega}_{\mathrm{X}, 1} t}\right)
\end{aligned}
$$




$$
\begin{aligned}
& +\left|x_{\mathrm{S}}\right\rangle\langle x x| \sqrt{2}\left(\mathcal{E}_{x} e^{-\mathrm{i} \tilde{\omega}_{\mathrm{X}, 1} t}+\mathcal{E}_{x}^{*} e^{\mathrm{i} \tilde{\omega}_{\mathrm{X}, 1} t}\right) \\
& +\left|y_{\mathrm{S}}\right\rangle\langle y y| \sqrt{2}\left(\mathcal{E}_{y} e^{-\mathrm{i} \tilde{\omega}_{\mathrm{Y}, 2} t}+\mathcal{E}_{y}^{*} e^{\mathrm{i} \tilde{\omega}_{\mathrm{Y}, 2} t}\right)+\text { H.c. }
\end{aligned}
$$

If we further assume that $\delta<\left|\omega_{\mathrm{X}, 1}-\omega_{\mathrm{X}, 2}\right|,\left|\omega_{\mathrm{Y}, 1}-\omega_{\mathrm{Y}, 2}\right|$, the first two lines of Eq. (14) represent a drive that is nearly resonant with the respective electronic transitions, whereas the remaining lines are off resonant. Furthermore, we assume that the sum of the drive frequencies is resonant with the two-photon transition from the ground state $|g\rangle$ to the doubly excited state $\left|x y_{\mathrm{S}}\right\rangle\left(\tilde{\omega}_{\mathrm{X}, 1}+\tilde{\omega}_{\mathrm{Y}, 2}=\omega_{\mathrm{X}, 1}+\omega_{\mathrm{Y}, 2}\right)$. In this case it is possible to apply the rotating-wave approximation and neglect the off-resonant terms:

$$
\frac{H_{\text {drive }}}{\hbar} \approx|g\rangle\left\langle x_{\mathrm{S}}\left|\sqrt{2} \mathcal{E}_{x}^{*} e^{\mathrm{i} \tilde{\omega}_{\mathrm{X}, 1} t}+\right| x_{\mathrm{S}}\right\rangle\left\langle x y_{\mathrm{S}}\right| \mathcal{E}_{y}^{*} e^{\mathrm{i} \tilde{\omega}_{\mathrm{Y}, 2} t}+\text { H.c. }
$$

We derive the effective Hamiltonian of the driven system by first considering the dynamics of a trial wave function:

$$
\left|\psi_{\text {drive }}\right\rangle=a^{g}|g\rangle+a^{x_{\mathrm{S}}}\left|x_{\mathrm{S}}\right\rangle+a^{y_{\mathrm{S}}}\left|y_{\mathrm{S}}\right\rangle+a^{x y_{\mathrm{S}}}\left|x y_{\mathrm{S}}\right\rangle,
$$

under the Hamiltonian in Eq. (15) expressed in the interaction picture with respect to the Hamitonian of the bare system (neglecting the small broadening due to spontaneous emission for the purpose of this derivation):

$$
\frac{H_{\text {sys }}}{\hbar}=\omega_{\mathrm{X}, 1}\left|x_{\mathrm{S}}\right\rangle\left\langle x_{\mathrm{S}}\left|+\omega_{\mathrm{Y}, 1}\right| y_{\mathrm{S}}\right\rangle\left\langle y_{\mathrm{S}}\left|+\omega_{x y_{\mathrm{S}}}\right| x y_{\mathrm{S}}\right\rangle\left\langle x y_{\mathrm{S}}\right| .
$$

The following differential equations can be obtained:

$$
\begin{aligned}
\dot{a}^{g}= & -i \sqrt{2} \mathcal{E}_{x}^{*} e^{i \delta t} a^{x_{\mathrm{S}}} \\
- & i \sqrt{2} \mathcal{E}_{y}^{*} e^{-i\left(\omega_{\mathrm{Y}, 1}-\tilde{\omega}_{\mathrm{Y}, 2}\right) t} a^{y_{\mathrm{S}}}, \\
\dot{a}^{x_{\mathrm{S}}}= & -i \sqrt{2} \mathcal{E}_{x} e^{i\left(\omega_{\mathrm{X}, 1}-\tilde{\omega}_{\mathrm{X}, 1}\right) t} a^{g} \\
& -i \mathcal{E}_{y}^{*} e^{-i\left(\omega_{\mathrm{Y}, 2}-\tilde{\omega}_{\mathrm{Y}, 2}\right) t} a^{x y_{\mathrm{S}}}, \\
\dot{a}^{y_{\mathrm{S}}}= & -i \sqrt{2} \mathcal{E}_{y} e^{i\left(\omega_{\mathrm{Y}, 1}-\tilde{\omega}_{\mathrm{Y}, 2}\right) t} a^{g} \\
& -i \mathcal{E}_{x}^{*} e^{-i\left(\omega_{\mathrm{X}, 2}-\tilde{\omega}_{\mathrm{X}, 1}\right) t} a^{x y_{\mathrm{S}}}, \\
\dot{a}^{x y_{\mathrm{S}}}= & -i \mathcal{E}_{x} e^{i\left(\omega_{\mathrm{X}, 2}-\tilde{\omega}_{\mathrm{X}, 1}\right) t} a^{y_{\mathrm{S}}} \\
& -i \mathcal{E}_{y} e^{i \delta t} a^{x_{\mathrm{S}}} .
\end{aligned}
$$

Equations (19) and (20) can be used to eliminate $a^{x_{\mathrm{S}}}$ and $a^{y_{\mathrm{S}}}$ in the adiabatic approximation:

$$
\begin{aligned}
a^{x_{\mathrm{S}}} \approx & \frac{\sqrt{2} \mathcal{E}_{x} a^{g}+\mathcal{E}_{y}^{*} a^{x y_{\mathrm{S}}}}{\delta} e^{-i \delta t}, \\
a^{y_{\mathrm{S}}} \approx & \frac{\sqrt{2} \mathcal{E}_{y}}{\tilde{\omega}_{\mathrm{Y}, 2}-\omega_{\mathrm{Y}, 1}} e^{-i\left(\tilde{\omega}_{\mathrm{Y}, 2}-\omega_{\mathrm{Y}, 1}\right) t} a^{g} \\
& +\frac{\mathcal{E}_{x}^{*}}{\omega_{\mathrm{X}, 2}-\tilde{\omega}_{\mathrm{X}, 1}} e^{-i\left(\omega_{\mathrm{X}, 2}-\tilde{\omega}_{\mathrm{X}, 1}\right) t} a^{x y_{\mathrm{S}}} .
\end{aligned}
$$

Eqs. (22) and (23) can be inserted into Eqs. (18) and (21). Neglecting rotating terms and small energy shifts, the effective dynamics are

$$
\begin{aligned}
& \dot{a}^{g}=-i g_{\mathrm{eff}} a^{x y_{\mathrm{s}},} \\
& \dot{a}^{x y_{\mathrm{s}}}=-i g_{\mathrm{eff}}^{*} a^{g},
\end{aligned}
$$

which correspond to the effective Hamiltonian

$$
H_{\text {drive }}^{\text {eff }} \approx \hbar g_{\text {eff }}|g\rangle\left\langle x y_{\mathrm{S}}\right|+\text { H.c., }
$$

with

$$
g_{\text {eff }}=\frac{\sqrt{2} \mathcal{E}_{x}^{*} \mathcal{E}_{y}^{*}}{\delta} .
$$

This Hamiltonian induces Rabi oscillations between $|g\rangle$ and $\left|x y_{\mathrm{S}}\right\rangle$ with frequency $2\left|g_{\text {eff }}\right|$. If the illumination is applied for time $\tau_{\text {drive }}=\pi /\left(2\left|g_{\text {eff }}\right|\right)$, the system is driven from the ground state to the desired state $\left|x y_{\mathrm{S}}\right\rangle$. An analogous pumping scheme exploiting the state $\left|y_{\mathrm{S}}\right\rangle$ with two lasers of polarizations and amplitudes $\mathcal{E}_{x} \hat{x}$ and $\mathcal{E}_{y} \hat{y}$, and respective frequencies $\tilde{\omega}_{\mathrm{X}, 2}=\omega_{\mathrm{X}, 2}-\delta$ and $\tilde{\omega}_{\mathrm{Y}, 1}=\omega_{\mathrm{Y}, 1}+\delta$, could be used to drive the system into the doubly excited state as well. Lastly, we remark that optimizing pumping schemes for state preparation of highly excited states, such as the $\left|x y_{\mathrm{s}}\right\rangle$ state that account for phonon-based dephasing and phonon-assisted transitions, is a complex and active area of research, as evidenced in the field of semiconducting quantum dots for deterministic generation of entangled photon pairs [43-52].

\section{CONCLUSIONS}

The present study provides the theoretical basis for a deterministic entangled photon-pair source from dipole-coupled emitters. Specifically, we dipole couple two three-level emitter systems, each with excited states with orthogonal transition dipole moments, to form a composite emitter system. When the composite emitter system is excited to a symmetric doubly excited state and subsequently deexcites in a radiative cascade, two entangled photons are emitted. We find that the entanglement measures of the emitted photons are robust to relative differences in frequency between the intermediate states. Importantly, the Bell state fidelity $\mathcal{F}$ and efficiency $\eta$ can be optimized, for example, by tuning the defect transition dipole moments.

The proposed scheme is especially amenable to defect emitters, although quantum dots or fixed molecules may be used to realize the scheme as well. Defects in both 2D and 3D have wide applicability in quantum technologies, especially as quantum memories because they combine the favorable coherence and nonclassical emission properties of isolated atoms $[53,54]$ with the scalability and stability of solid-state technologies [55-58]. A key breakthrough that highlights their applicability is the experimental demonstration of memory-enhanced quantum communication for quantum repeaters [59].

Defects are natural candidates because of their fixed geometries enabling stable dipole coupling, diverse symmetries that allow well defined and orthogonal transition dipole moments, and emission properties that can be tailored chemically or externally and also integrated on-chip for a variety of quantum technologies. In addition, the chemical selection space of defect systems is vast, as the chemical identity of the defect and surrounding matrix can be permuted to discover the appropriate system for a specific application [60]. Because accurately computing multiple excited states remains a significant challenge [61], the present scheme involving just singly excited states is more amenable to computational searches 
TABLE II. The dipole operator $\boldsymbol{d}$ in the eigenbasis.

\begin{tabular}{lcc}
\hline \hline Initial & Final & $\boldsymbol{d}$ \\
\hline$|g\rangle$ & $\left|x_{\mathrm{S}}\right\rangle$ & $\sqrt{2} d_{x} \hat{x}$ \\
$|g\rangle$ & $\left|y_{\mathrm{S}}\right\rangle$ & $\sqrt{2} d_{y} \hat{y}$ \\
$\left|x_{\mathrm{S}}\right\rangle$ & $\left|x y_{\mathrm{S}}\right\rangle$ & $d_{y} \hat{y}$ \\
$\left|y_{\mathrm{S}}\right\rangle$ & $\left|x y_{\mathrm{S}}\right\rangle$ & $d_{x} \hat{x}$ \\
$\left|x_{\mathrm{S}}\right\rangle$ & $|x x\rangle$ & $\sqrt{2} d_{x} \hat{x}$ \\
$\left|y_{\mathrm{S}}\right\rangle$ & $|y y\rangle$ & $\sqrt{2} d_{y} \hat{y}$ \\
$\left|x_{\mathrm{A}}\right\rangle$ & $\left|x y_{\mathrm{A}}\right\rangle$ & $d_{y} \hat{y}$ \\
$\left|y_{\mathrm{A}}\right\rangle$ & $\left|x y_{\mathrm{A}}\right\rangle$ & $d_{x} \hat{x}$ \\
\hline \hline
\end{tabular}

of defect system candidates. A current challenge of realizing defect-based quantum emitters, however, is the relatively low phonon-limited quantum efficiency, the highest of which has been observed to be $87 \% \pm 7 \%$ for single-photon emitters in hBN [62] as compared to theoretical predictions of $>96 \%$ phonon-limited quantum efficiency in semiconductor quantum dots with realistic experimental parameters [63]. As is the case in semiconductor quantum dots [33,49,64-66], system imperfections of defect-based systems may be modulated by coupling defects to external fields, including electric, magnetic, and strain, as well as to waveguides and sculpted electromagnetic environments of cavities to improve fidelity and collection efficiency. Several of these effects have been studied extensively in defect systems [67-72], thereby enabling near-term experimental observations of the present proposal.

\section{ACKNOWLEDGMENTS}

We acknowledge fruitful discussions with Christopher Ciccarino, Stefan Krastanov, Matthew Trusheim, and Dirk Englund. This work was supported by the Department of Energy 'Photonics at Thermodynamic Limits' Energy Frontier Research Center under grant DE-SC0019140. T.N. is partially supported by the U.S. Department of Energy, Office of Science, Basic Energy Sciences (BES), Materials Sciences and Engineering Division under FWP ERKCK47 'Understanding and Controlling Entangled and Correlated Quantum States in Confined Solid-state Systems Created via Atomic Scale Manipulation'. D.S.W. is an NSF Graduate Research Fellow. P.N. is a Moore Inventor Fellow through Grant GBMF8048 from the Gordon and Betty Moore Foundation.

\section{APPENDIX A: DIPOLE OPERATOR}

We explicitly write the dipole operator in the eigenbasis of the total electronic Hamiltonian $H_{\mathrm{el}}$ in Table II.

\section{APPENDIX B: WEISSKOPF-WIGNER APPROXIMATION}

Here we explicitly show how we obtain Eq. (7) in the main text, the wave function coefficient of the steady state electronphoton state. We reproduce the ansatz for a general electronphoton wave function from Eq. (5) in the main text:

$$
\begin{aligned}
|\Psi(t)\rangle= & \sum_{j k} c_{j k}^{g}|g\rangle a_{j X}^{\dagger} a_{k Y}^{\dagger}|\mathrm{vac}\rangle+\sum_{j} c_{j}^{x_{\mathrm{S}}}\left|x_{\mathrm{S}}\right\rangle a_{j Y}^{\dagger}|\mathrm{vac}\rangle \\
& +\sum_{j} c_{j}^{y_{\mathrm{S}}}\left|y_{\mathrm{S}}\right\rangle a_{j X}^{\dagger}|\mathrm{vac}\rangle+c^{x y_{\mathrm{S}}}\left|x y_{\mathrm{S}}\right\rangle|\mathrm{vac}\rangle
\end{aligned}
$$

The interaction Hamiltonian is:

$$
\begin{aligned}
H_{\mathrm{int}}= & \sum_{j} \Omega_{y_{\mathrm{S}}, x y_{\mathrm{S}}}\left|y_{\mathrm{S}}, 1_{j X}, 0_{k Y}\right\rangle\left\langle x y_{\mathrm{S}}\right|+\text { H.c. } \\
& +\sum_{j} \Omega_{x_{\mathrm{S}}, x y_{\mathrm{S}}}\left|x_{\mathrm{S}}, 0_{k X}, 1_{j Y}\right\rangle\left\langle x y_{\mathrm{S}}\right|+\text { H.c. } \\
& +\sum_{j k} \Omega_{g, y_{\mathrm{S}}}\left|g, 1_{j X}, 1_{k Y}\right\rangle\left\langle y_{\mathrm{S}}, 1_{j X}, 0_{k Y}\right|+\text { H.c. } \\
& +\sum_{j k} \Omega_{g, x_{\mathrm{S}}}\left|g, 1_{j X}, 1_{k Y}\right\rangle\left\langle x_{\mathrm{S}}, 0_{j X}, 1_{k Y}\right|+\text { H.c. }
\end{aligned}
$$

We now plug this state vector into the Schrödinger equation to derive the differential equations for the coefficients:

$$
\frac{d}{d t} c^{x y_{\mathrm{S}}}=-i \omega_{x y_{\mathrm{S}}} c^{x y_{\mathrm{S}}}-i \sum_{j} \Omega_{y_{\mathrm{S}}, x y_{\mathrm{S}}} c_{j}^{y_{\mathrm{S}}}-i \sum_{j} \Omega_{x_{\mathrm{S}}, x y_{\mathrm{S}}} c_{j}^{x_{\mathrm{S}}},
$$

$$
\frac{d}{d t} c_{j}^{x_{\mathrm{S}}}=-i\left(\omega_{x_{\mathrm{S}}}+\omega_{j}\right) c_{j}^{x_{\mathrm{S}}}-i \Omega_{x_{\mathrm{S}}, x y_{\mathrm{S}}} c^{x y_{\mathrm{S}}}-i \sum_{k} \Omega_{g, x_{\mathrm{S}}} c_{j k}^{g},
$$

$$
\begin{gathered}
\frac{d}{d t} c_{j}^{y_{\mathrm{S}}}=-i\left(\omega_{y_{\mathrm{S}}}+\omega_{j}\right) c_{j}^{y_{\mathrm{S}}}-i \Omega_{y_{\mathrm{S}}, x y_{\mathrm{S}}} c^{x y_{\mathrm{S}}}-i \sum_{k} \Omega_{g, y_{\mathrm{S}}} c_{j k}^{g}, \\
\frac{d}{d t} c_{j k}^{g}=-i\left(\omega_{j}+\omega_{k}\right) c_{j k}^{g}-i \Omega_{g, y_{\mathrm{S}}} c_{j}^{y_{\mathrm{S}}}-i \Omega_{g, x_{\mathrm{S}}} c_{j}^{x_{\mathrm{S}}},
\end{gathered}
$$

where we assume $\Omega_{o p}$ is real. We now solve the differential equations in the Weisskopf-Wigner approximation. We first take Eq. (B4) and formally integrate it:

$$
\begin{aligned}
c_{j}^{x_{\mathrm{S}}}= & c_{j}^{x_{\mathrm{S}}}(0) e^{-i\left(\omega_{x_{\mathrm{S}}}+\omega_{k}\right) t}-i \Omega_{x_{\mathrm{S}}, x y_{\mathrm{S}}} \int_{0}^{t} e^{-i\left(\omega_{x_{\mathrm{S}}}+\omega_{k}\right)(t-\tau)} c^{x y_{\mathrm{S}}}(\tau) d \tau \\
& -i \Omega_{g, x_{\mathrm{S}}} \int_{0}^{t} e^{-i\left(\omega_{x_{\mathrm{S}}}+\omega_{k}\right)(t-\tau)} c_{j k}^{g}(\tau) d \tau .
\end{aligned}
$$

We get an analogous equation for $c_{j}^{y_{\mathrm{S}}}$ and insert both into Eq. (B3):

$$
\begin{aligned}
\frac{d}{d t} c^{x y_{\mathrm{S}}} & =-i \omega_{x y_{\mathrm{S}}} c^{x y_{\mathrm{S}}}-i \sum_{j} \Omega_{y_{\mathrm{S}}, x y_{\mathrm{S}}}\left(-i \Omega_{y_{\mathrm{S}}, x y_{\mathrm{S}}} \int_{0}^{t} e^{-i\left(\omega_{y_{\mathrm{S}}}+\omega_{k}\right)(t-\tau)} c^{x y_{\mathrm{S}}}(\tau) d \tau-i \Omega_{g, y_{\mathrm{S}}} \int_{0}^{t} e^{-i\left(\omega_{y_{\mathrm{S}}}+\omega_{k}\right)(t-\tau)} c_{j k}^{g}(\tau) d \tau\right) \\
& -i \sum_{j} \Omega_{x, x y_{\mathrm{S}}}\left(-i \Omega_{x_{\mathrm{S}}, x y_{\mathrm{S}}} \int_{0}^{t} e^{-i\left(\omega_{x_{\mathrm{S}}}+\omega_{k}\right)(t-\tau)} c^{x y_{\mathrm{S}}}(\tau) d \tau-i \Omega_{g, x_{\mathrm{S}}} \int_{0}^{t} e^{-i\left(\omega_{x_{\mathrm{S}}}+\omega_{k}\right)(t-\tau)} c_{j k}^{g}(\tau) d \tau\right),
\end{aligned}
$$


In the Weisskopf-Wigner approximation it is commonly assumed that the time integrals can be extended to infinity and that the $\tau$ dependent coefficients can be extracted from the integral by setting $\tau=t$. Since we are operating in the Schrödinger picture, we have to perform this procedure with caution and define the slowly-varying amplitudes of a coefficient $c^{A}(\tau)=e^{-i \omega_{A} \tau} \tilde{c}^{A}(\tau)$. We then set $\tilde{c}^{A}(\tau) \approx \tilde{c}^{A}(t)$, which is equivalent to performing the Markov approximation in the interaction picture. In this approximation we get:

$$
\begin{aligned}
& -\sum_{j}\left|\Omega_{y_{\mathrm{S}}, x y_{\mathrm{S}}}\right|^{2} \int_{0}^{t} e^{-i\left(\omega_{y_{\mathrm{S}}}+\omega_{k}\right)(t-\tau)} e^{-i \omega_{x y_{\mathrm{S}}} \tau} \tilde{c}^{x y_{\mathrm{S}}}(\tau) d \tau \\
& \approx-\sum_{j}\left|\Omega_{y_{\mathrm{S}}, x y_{\mathrm{S}}}\right|^{2} \tilde{c}^{x y_{\mathrm{S}}}(t) \int_{0}^{t} e^{-i\left(\omega_{y_{\mathrm{S}}}+\omega_{k}\right)(t-\tau)} e^{-i \omega_{x y_{\mathrm{S}}} \tau} d \tau .
\end{aligned}
$$

The integral in the last line can be further decomposed and the lower integration limit can be extended to $-\infty$ :

$$
\begin{aligned}
& e^{-i\left(\omega_{y_{\mathrm{S}}}+\omega_{k}\right) t} \int_{-\infty}^{t} e^{-i\left(\omega_{x y_{\mathrm{S}}}-\omega_{y_{\mathrm{S}}}-\omega_{j}\right) \tau} d \tau \\
& \approx e^{-i \omega_{x y_{\mathrm{S}}} t}\left(\pi \delta\left(\omega_{x y_{\mathrm{S}}}-\omega_{y_{\mathrm{S}}}-\omega_{j}\right)+i \mathrm{P}\left\{\frac{1}{\omega_{x y_{\mathrm{S}}}-\omega_{y_{\mathrm{S}}}-\omega_{j}}\right\}\right) .
\end{aligned}
$$

We further neglect the imaginary part of the parenthesis, the principal part $(\mathrm{P}\{\})$ that generally leads to a spectral shift, and we retain only the delta function. We note that, in the discrete case, $\delta\left(\omega_{k}-\omega_{j}\right) \rightarrow \delta_{j k} / \Delta$ (which is a discrete representation of the delta function). Notice also that $e^{-i \omega_{x y_{\mathrm{S}}} t} \tilde{c}^{x y_{\mathrm{S}}}(t)=c^{x y_{\mathrm{S}}}(t)$. We therefore get the result:

$$
\begin{gathered}
-\sum_{j}\left|\Omega_{y_{\mathrm{S}}, x y_{\mathrm{S}}}\right|^{2} \int_{0}^{t} e^{-i\left(\omega_{y_{\mathrm{S}}}+\omega_{k}\right)(t-\tau)} c^{x y_{\mathrm{S}}}(\tau) d \tau \\
\approx-\frac{\pi\left|\Omega_{y_{\mathrm{S}}, x y_{\mathrm{S}}}\right|^{2}}{\Delta} c^{x y_{\mathrm{S}}}(t) \equiv-\gamma_{y_{\mathrm{S}}, x y_{\mathrm{S}}} c^{x y_{\mathrm{S}}}(t) .
\end{gathered}
$$

We get a similar result for the first term in the second parenthesis of Eq. (B8):

$$
\approx-\gamma_{x_{\mathrm{S}}, x y_{\mathrm{S}}} c^{x y_{\mathrm{S}}}(t)
$$

The remaining terms in Eq. (B8) yield after applying the same procedure:

$$
\begin{gathered}
-\pi \sum_{j}\left[\Omega_{y_{\mathrm{S}}, x y_{\mathrm{S}}} \Omega_{g, y_{\mathrm{S}}} c_{j k}^{g}(t) \delta\left(\omega_{k}-\omega_{y_{\mathrm{S}}}\right)\right. \\
\left.+\Omega_{x_{\mathrm{S}}, x y_{\mathrm{S}}} \Omega_{g, x_{\mathrm{S}}} c_{k j}^{g}(t) \delta\left(\omega_{k}-\omega_{x_{\mathrm{S}}}\right)\right] .
\end{gathered}
$$

This term is neglected in the calculations because of the frequency restriction imposed by the delta function, although in principle this term is of the same order as the terms leading to decay. We therefore obtain:

$$
\frac{d}{d t} c^{x y_{\mathrm{S}}}=-i \omega_{x y_{\mathrm{S}}} c^{x y_{\mathrm{S}}}-\left(\gamma_{x_{\mathrm{S}}, x y_{\mathrm{S}}}+\gamma_{y_{\mathrm{S}}, x y_{\mathrm{S}}}\right) c^{x y_{\mathrm{S}}} .
$$

Similarly, we can derive the remaining differential equations:

$$
\frac{d}{d t} c_{j}^{x_{\mathrm{S}}}=-\mathrm{i}\left(\omega_{x_{\mathrm{S}}}+\omega_{j}\right) c_{j}^{x_{\mathrm{S}}}-\gamma_{g, x_{\mathrm{S}}} c_{j}^{x_{\mathrm{S}}}-\mathrm{i} \Omega_{x_{\mathrm{S}}, x y_{\mathrm{S}}} c^{x y_{\mathrm{S}}},
$$
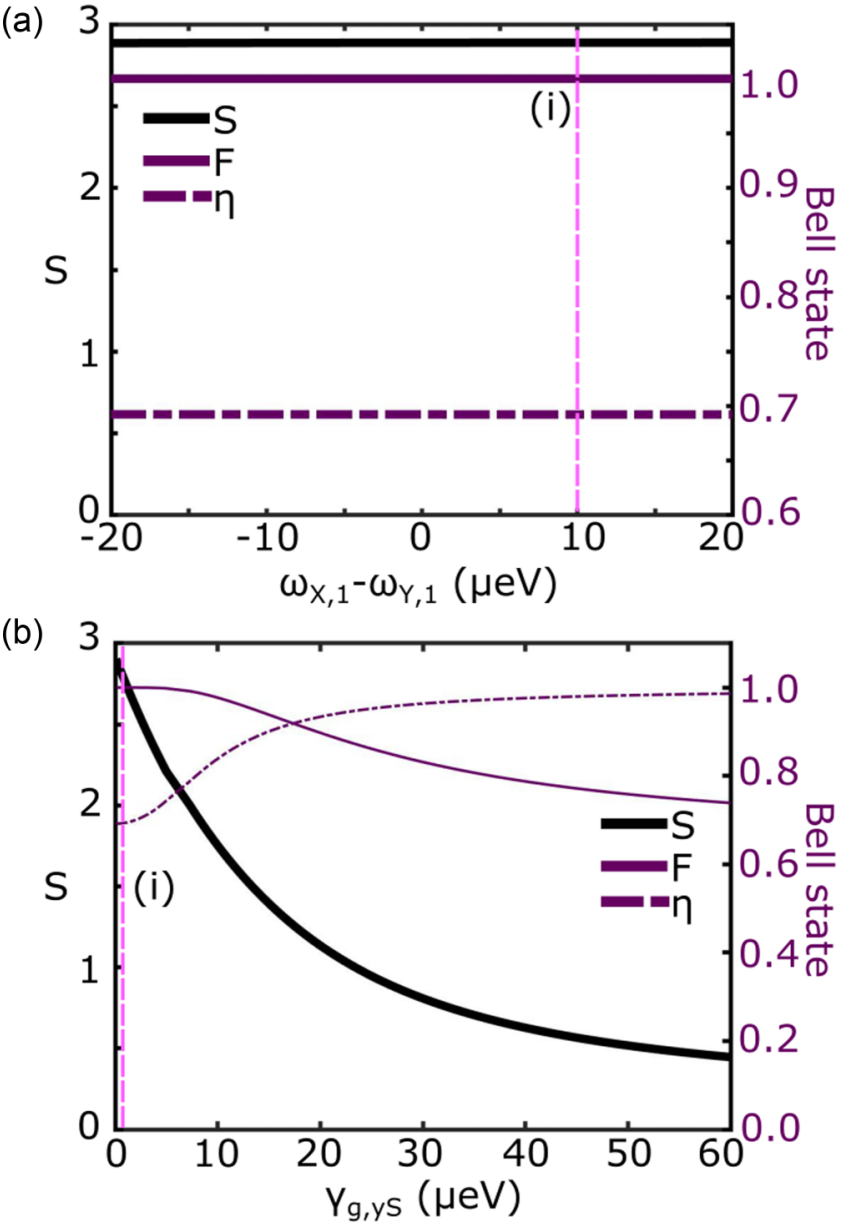

FIG. 4. Entanglement entropy $S$, Bell state fidelity $\mathcal{F}$, and Bell state efficiency $\eta$ are (a) unaffected by varying $\omega_{X, 1}$ and (b) impacted by increasing the emission line width. The pink line (i) corresponds to the conditions in Fig. 2 of the main text.

$$
\begin{gathered}
\frac{d}{d t} c_{j}^{y_{\mathrm{S}}}=-\mathrm{i}\left(\omega_{y_{\mathrm{S}}}+\omega_{j}\right) c_{j}^{y_{\mathrm{S}}}-\gamma_{g, y_{\mathrm{S}}} c_{j}^{y_{\mathrm{S}}}-\mathrm{i} \Omega_{y_{\mathrm{S}}, x y_{\mathrm{S}}} c^{x y_{\mathrm{S}}}, \\
\frac{d}{d t} c_{j k}^{g}=-\mathrm{i}\left(\omega_{j}+\omega_{k}\right) c_{j k}^{g}-\mathrm{i} \Omega_{g, y_{\mathrm{S}}} c_{j}^{y_{\mathrm{S}}}-\mathrm{i} \Omega_{g, x_{\mathrm{S}}} c_{k}^{x_{\mathrm{S}}} .
\end{gathered}
$$

This system of equations can be solved with the initial conditions:

$$
\begin{aligned}
c^{x y_{\mathrm{S}}}(0) & =1, \\
c_{j}^{x_{\mathrm{S}}}(0) & =c_{j}^{y_{\mathrm{S}}}(0)=c_{j k}^{g}(0)=0,
\end{aligned}
$$

with the following steady-state solution in the rotating frame:

$$
\tilde{c}_{j k}^{g}(\infty)=\frac{\frac{-\Omega_{g, x_{\mathrm{S}}} \Omega_{x_{\mathrm{S}}, x_{\mathrm{S}}}}{i \omega_{x_{\mathrm{S}}}-i \omega_{j}+\gamma_{g, x_{\mathrm{S}}}}+\frac{-\Omega_{g, y_{\mathrm{S}}} \Omega_{y_{\mathrm{S}}, x_{\mathrm{S}}}}{i \omega_{y_{\mathrm{S}}}-i \omega_{k}+\gamma_{g, y_{\mathrm{S}}}}}{i\left(\omega_{x y_{\mathrm{S}}}-\omega_{j}-\omega_{k}\right)+\gamma_{x_{\mathrm{S}}, x y_{\mathrm{S}}}+\gamma_{y_{\mathrm{S}}, x y_{\mathrm{S}}}},
$$

which matches Eq. (7) in the main text.

\section{APPENDIX C: ROBUST ENTANGLEMENT}

In Fig. 4, we show that the entanglement of the emitted photon pair is robust to changes in $\omega_{X, 1}$ relative to $\omega_{Y, 1}$, while $\mathcal{F}$ and $S$ decrease as $\gamma_{g, y_{S}}$ increases. 


\section{APPENDIX D: DEPHASING}

We consider the impact of emitter imperfections, such as phonon-based dephasing of defect emitters, that results in fluctuations in the energies of the defect emitters. We implement the effect of dephasing by averaging the final states of the emitted photons over an ensemble of quantum states generated using a probabilty distribution of emitter frequencies, reflecting the broadening of the transition frequencies due to dephasing effects [73]. The fidelity $\mathcal{F}_{\text {de }}$ in the presence of dephasing can be estimated as

$$
\begin{gathered}
\mathcal{F}_{\mathrm{de}}=\iiint\left[d \omega_{x_{\mathrm{S}}}^{0} d \omega_{y_{\mathrm{S}}}^{0} d \omega_{x y_{\mathrm{S}}}^{0} P\left(\omega_{x_{\mathrm{S}}}^{0}, \omega_{y_{\mathrm{S}}}^{0}, \omega_{x y_{\mathrm{S}}}^{0}, \sigma\right)\right. \\
\left.\left|\left\langle\psi_{\mathrm{de}}\left(\omega_{x_{\mathrm{S}}}^{0}, \omega_{y_{\mathrm{S}}}^{0}, \omega_{x y_{\mathrm{S}}}^{0}\right) \mid \Psi^{+}\right\rangle\right|^{2}\right]
\end{gathered}
$$

where $\left|\psi_{\mathrm{de}}\left(\omega_{x_{\mathrm{S}}}, \omega_{y_{\mathrm{S}}}, \omega_{x y_{\mathrm{S}}}\right)\right\rangle$ is calculated just as $|\psi\rangle$ is in Eq. (11), except that the central frequencies of the emitters $\omega_{i}$ are substituted by $\omega_{i}^{0}$ in Eq. (7), where $i \in\left\{x_{\mathrm{S}}, y_{\mathrm{S}}, x y_{\mathrm{S}}\right\}$. Explicitly, $|\psi\rangle=\left|\psi_{\mathrm{de}}\left(\omega_{x_{\mathrm{S}}}, \omega_{y_{\mathrm{S}}}, \omega_{x y_{\mathrm{S}}}\right)\right\rangle$, as in Eq. (11). We assume that $\omega_{x_{\mathrm{S}}}^{0}, \omega_{y_{\mathrm{S}}}^{0}$, and $\omega_{x y_{\mathrm{S}}}$ belong to a probability distribution $P$. While the exact probability distribution depends on the microscopic physical mechanism underlying dephasing, we choose to represent $P$ as a 3D Gaussian [74] $\prod_{i} G_{i}\left(\Delta \omega_{i}, \sigma\right)$, physically representing independent fluctuations of the energy levels of the relevant excited states following a Gaussian distribution. We plot the fidelity in the presence of phonon-based dephasing in Fig. 5, where $\sigma_{i}$ is the full width at half maximum, representing the dephasing rate and $\Delta \omega_{i}=\omega_{i}^{0}-\omega_{i}$.

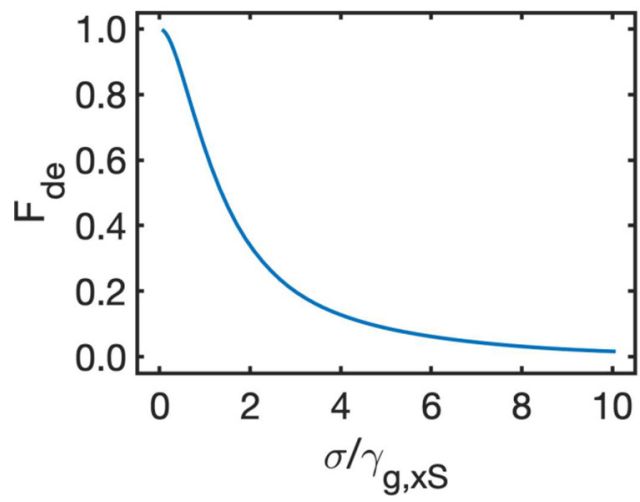

FIG. 5. Fidelity $\mathcal{F}_{\text {de }}$ in the presence of Gaussian dephasing with dephasing rate $\sigma$, normalized by the radiative rate $\gamma_{g, x S}$, for the conditions corresponding to the pink lines in Figs. 3 and 4.

The fidelity in the presence of dephasing $\mathcal{F}_{\text {de }}$ is limited by the radiative linewidth $\gamma_{g, x S}$, suggesting ideal operation in dilution fridges for the rates described here. Lastly, we note that while we have considered the effect of phonon-based dephasing on the performance of the presently described scheme, we have not considered phonon-assisted transitions between, for instance, symmetric and antisymmetric states in the composite energy diagram in Fig. 1. These effects are known to have a major impact on the resulting collection efficiency of entangled photon pairs from the biexciton decay cascade of semiconducting quantum dots, as described in Sec. IV, and warrant further investigation upon selection of a candidate defect system.
[1] J. L. O’Brien, A. Furusawa, and J. Vučković, Photonic quantum technologies, Nat. Photonics 3, 687 (2009).

[2] Y. Chen, J. Zhang, M. Zopf, K. Jung, Y. Zhang, R. Keil, F. Ding, and O. G. Schmidt, Wavelength-tunable entangled photons from silicon-integrated III-V quantum dots, Nat. Commun. 7, 10387 (2016).

[3] J. W. Pan, Z. B. Chen, C. Y. Lu, H. Weinfurter, A. Zeilinger, and M. Zukowski, Multiphoton entanglement and interferometry, Rev. Mod. Phys. 84, 777 (2012).

[4] P. C. Humphreys, N. Kalb, J. P. J. Morits, R. N. Schouten, R. F. L. Vermeulen, D. J. Twitchen, M. Markham, and R. Hanson, Deterministic delivery of remote entanglement on a quantum network, Nature 558, 268 (2018).

[5] N. Sangouard, C. Simon, H. de Riedmatten, and N. Gisin, Quantum repeaters based on atomic ensembles and linear optics, Rev. Mod. Phys. 83, 33 (2011).

[6] V. Scarani, H. de Riedmatten, I. Marcikic, H. Zbinden, and N. Gisin, Four-photon correction in two-photon Bell experiments, Eur. Phys. J. D 32, 129 (2005).

[7] B. Zhao, M. Müller, K. Hammerer, and P. Zoller, Efficient quantum repeater based on deterministic Rydberg gates, Phys. Rev. A 81, 052329 (2010).

[8] P. G. Kwiat, S. Barraza-Lopez, A. Stefanov, and N. Gisin, Experimental entanglement distillation and 'hidden' non-locality, Nature 409, 1014 (2001).
[9] D. C. Burnham and D. L. Weinberg, Observation of Simultaneity in Parametric Production of Optical Photon Pairs, Phys. Rev. Lett. 25, 84 (1970).

[10] A. Anwar, C. Perumangatt, F. Steinlechner, T. Jennewein, and A. Ling, Entangled photon-pair sources based on three-wave mixing in bulk crystals, arXiv:2007.15364.

[11] H. Takesue and K. Inoue, Generation of polarization-entangled photon pairs and violation of bell's inequality using spontaneous four-wave mixing in a fiber loop, Phys. Rev. A 70, 031802(R) (2004).

[12] X. Lu, Q. Li, D. A. Westly, G. Moille, A. Singh, V. Anant, and K. Srinivasan, Chip-integrated visible-telecom entangled photon pair source for quantum communication, Nat. Phys. 15, 373 (2019).

[13] L. Lanco, S. Ducci, J. P. Likforman, X. Marcadet, J. A. W. van Houwelingen, H. Zbinden, G. Leo, and V. Berger, Semiconductor Waveguide Source of Counterpropagating Twin Photons, Phys. Rev. Lett. 97, 173901 (2006).

[14] J. C. Howell, R. S. Bennink, S. J. Bentley, and R. W. Boyd, Realization of the Einstein-Podolsky-Rosen Paradox Using Momentum and Position-Entangled Photons from Spontaneous Parametric Down Conversion, Phys. Rev. Lett. 92, 210403 (2004).

[15] R. Horn, P. Abolghasem, B. J. Bijlani, D. Kang, A. S. Helmy, and G. Weihs, Monolithic Source of Photon Pairs, Phys. Rev. Lett. 108, 153605 (2012). 
[16] E. Waks, E. Diamanti, B. C. Sanders, S. D. Bartlett, and Y. Yamamoto, Direct Observation of Nonclassical Photon Statistics in Parametric Down-Conversion, Phys. Rev. Lett. 92, 113602 (2004).

[17] N. Akopian, N. H. Lindner, E. Poem, Y. Berlatzky, J. Avron, D. Gershoni, B. D. Gerardot, and P. M. Petroff, Entangled Photon Pairs from Semiconductor Quantum Dots, Phys. Rev. Lett. 96, 130501 (2006).

[18] A. Muller, W. Fang, J. Lawall, and G. S. Solomon, Creating Polarization-Entangled Photon Pairs from a Semiconductor Quantum Dot Using the Optical Stark Effect, Phys. Rev. Lett. 103, 217402 (2009).

[19] A. Orieux, M. A. M. Versteegh, K. D. Jöns, and S. Ducci, Semiconductor devices for entangled photon pair generation: A review, Rep. Prog. Phys. 80, 076001 (2017).

[20] D. Huber, M. Reindl, J. Aberl, A. Rastelli, and R. Trotta, Semiconductor quantum dots as an ideal source of polarizationentangled photon pairs on-demand: A review, J. Opt. 20, 073002 (2018).

[21] M. Zeeshan, N. Sherlekar, A. Ahmadi, R. L. Williams, and M. E. Reimer, Proposed Scheme to Generate Bright Entangled Photon Pairs by Application of a Quadrupole Field to a Single Quantum Dot, Phys. Rev. Lett. 122, 227401 (2019).

[22] J. Liu, R. Su, Y. Wei, B. Yao, S. F. C. da Silva, Y. Yu, J. Iles-Smith, K. Srinivasan, A. Rastelli, J. Li, and X. Wang, A solid-state source of strongly entangled photon pairs with high brightness and indistinguishability, Nat. Nanotechnol. 14, 586 (2019).

[23] A. Fognini, A. Ahmadi, M. Zeeshan, J. T. Fokkens, S. J. Gibson, N. Sherlekar, S. J. Daley, D. Dalacu, P. J. Poole, K. D. Jöns, V. Zwiller, and M. E. Reimer, Dephasing free photon entanglement with a quantum dot, ACS Photonics 6, 1656 (2019).

[24] A. Ahmadi, A. Fognini, and M. E. Reimer, Toward on-demand generation of entangled photon pairs with a quantum dot, in Recent Advances in Nanophotonics-Fundamentals and Applications (IntechOpen Limited, London, UK, 2020).

[25] M. D. Lukin and P. R. Hemmer, Quantum Entanglement Via Optical Control Of Atom-Atom Interactions, Phys. Rev. Lett. 84, 2818 (2000).

[26] N. L. McDougall, J. G. Partridge, R. J. Nicholls, S. P. Russo, and D. G. McCulloch, Influence of point defects on the near edge structure of hexagonal boron nitride, Phys. Rev. B 96, 144106 (2017).

[27] I. Harris, C. J. Ciccarino, J. Flick, D. R. Englund, and P. Narang, Group-III quantum defects in diamond are stable Spin-1 color centers, Phys. Rev. B 102, 195206 (2020).

[28] M. E. Trusheim, N. H. Wan, K. C. Chen, C. J. Ciccarino, J. Flick, R. Sundararaman, G. Malladi, E. Bersin, M. Walsh, B. Lienhard, H. Bakhru, P. Narang, and D. Englund, Leadrelated quantum emitters in diamond, Phys. Rev. B 99, 075430 (2019).

[29] F. Hayee, L. Yu, J. L. Zhang, C. J. Ciccarino, M. Nguyen, A. F. Marshall, I. Aharonovich, J. Vučković, P. Narang, T. F. Heinz, and J. A. Dionne, Revealing multiple classes of stable quantum emitters in hexagonal boron nitride with correlated optical and electron microscopy, Nat. Mater. 19, 534 (2020).

[30] C. J. Ciccarino, J. Flick, I. B. Harris, M. E. Trusheim, D. R. Englund, and P. Narang, Strong spin-orbit quenching via the product jahn-teller effect in neutral Group IV artificial atom qubits in diamond, npj Quantum Mater. 5, 75 (2020).
[31] D. Bhattacharya, N. Vaval, and S. Pal, Electronic transition dipole moments and dipole oscillator strengths within Fockspace multi-reference coupled cluster framework: An efficient and novel approach, J. Chem. Phys. 138, 094108 (2013).

[32] V. Weisskopf and E. Wigner, Berechnung der natürlichen Linienbreite auf Grund der Diracschen Lichttheorie, Z. Phys. 63, 54 (1930).

[33] P. K. Pathak and S. Hughes, Generation of entangled photon pairs from a single quantum dot embedded in a planar photoniccrystal cavity, Phys. Rev. B 79, 205416 (2009).

[34] S. Parker, S. Bose, and M. B. Plenio, Entanglement quantification and purification in continuous-variable systems, Phys. Rev. A 61, 032305 (2000).

[35] C. K. Law, I. A. Walmsley, and J. H. Eberly, Continuous Frequency Entanglement: Effective Finite Hilbert Space and Entropy Control, Phys. Rev. Lett. 84, 5304 (2000).

[36] H. Huang and J. H. Eberly, Correlations and one-quantum pulse shapes in photon pair generation, J. Mod. Optic. 40, 915 (1993).

[37] Z. Zhao, J.-W. Pan, and M. S. Zhan, Practical scheme for entanglement concentration, Phys. Rev. A 64, 014301 (2001).

[38] S. Bose, V. Vedral, and P. L. Knight, Purification via entanglement swapping and conserved entanglement, Phys. Rev. A 60 194 (1999).

[39] C. H. Bennett, H. J. Bernstein, S. Popescu, and B. Schumacher, Concentrating partial entanglement by local operations, Phys. Rev. A 53, 2046 (1996).

[40] S. Krastanov, V. V. Albert, and L. Jiang, Optimized entanglement purification, Quantum 3, 123 (2019).

[41] A. Sørensen and K. Mølmer, Quantum Computation with Ions in Thermal Motion, Phys. Rev. Lett. 82, 1971 (1999).

[42] Y. Chen, M. Zopf, R. Keil, F. Ding, and O. G. Schmidt, Highlyefficient extraction of entangled photons from quantum dots using a broadband optical antenna, Nat. Commun. 9, 2994 (2018).

[43] A. Carmele and S. Reitzenstein, Non-Markovian features in semiconductor quantum optics: Quantifying the role of phonons in experiment and theory, Nanophotonics 8, 655 (2019).

[44] J. Förstner, C. Weber, J. Danckwerts, and A. Knorr, PhononAssisted Damping of Rabi Oscillations in Semiconductor Quantum Dots, Phys. Rev. Lett. 91, 127401 (2003).

[45] A. Nazir and D. P. S. McCutcheon, Modelling exciton-phonon interactions in optically driven quantum dots, J. Phys. Condens. Matter 28, 103002 (2016).

[46] E. V. Denning, J. Iles-Smith, N. Gregersen, and J. Mork, Phonon effects in quantum dot single-photon sources, Opt. Mater. Express 10, 222 (2020).

[47] C. Gustin and S. Hughes, Pulsed excitation dynamics in quantum-dot-cavity systems: Limits to optimizing the fidelity of on-demand single-photon sources, Phys. Rev. B 98, 045309 (2018).

[48] J. H. Quilter, A. J. Brash, F. Liu, M. Glässl, A. M. Barth V. M. Axt, A. J. Ramsay, M. S. Skolnick, and A. M. Fox, Phonon-Assisted Population Inversion of a Single InGaAs/GaAs Quantum Dot by Pulsed Laser Excitation, Phys. Rev. Lett. 114, 137401 (2015).

[49] A. N. Poddubny, P. Ginzburg, P. A. Belov, A. V. Zayats, and Y. S. Kivshar, Tailoring and enhancing spontaneous two-photon emission using resonant plasmonic nanostructures, Phys. Rev. A 86, 033826 (2012). 
[50] A. J. Ramsay, T. M. Godden, S. J. Boyle, E. M. Gauger, A. Nazir, B. W. Lovett, A. M. Fox, and M. S. Skolnick, PhononInduced Rabi-Frequency Renormalization of Optically Driven Single InGaAs/GaAs Quantum Dots, Phys. Rev. Lett. 105, 177402 (2010).

[51] S. M. Ulrich, S. Ates, S. Reitzenstein, A. Löffler, A. Forchel, and P. Michler, Dephasing of Triplet-Sideband Optical Emission of a Resonantly Driven InAs/GaAs Quantum Dot Inside a Microcavity, Phys. Rev. Lett. 106, 247402 (2011).

[52] S. Weiler, A. Ulhaq, S. M. Ulrich, D. Richter, M. Jetter, P. Michler, C. Roy, and S. Hughes, Phonon-assisted incoherent excitation of a quantum dot and its emission properties, Phys. Rev. B 86, 241304(R) (2012).

[53] C. Kurtsiefer, S. Mayer, P. Zarda, and H. Weinfurter, Stable Solid-State Source of Single Photons, Phys. Rev. Lett. 85, 290 (2000).

[54] G. Grosso, H. Moon, B. Lienhard, S. Ali, D. K. Efetov, M. M. Furchi, P. Jarillo-Herrero, M. J. Ford, I. Aharonovich, and D. Englund, Tunable and high-purity room temperature single-photon emission from atomic defects in hexagonal boron nitride, Nat. Commun. 8, 705 (2017).

[55] C. L. Degen, F. Reinhard, and P. Cappellaro, Quantum sensing, Rev. Mod. Phys. 89, 35002 (2017).

[56] I. Aharonovich, D. Englund, and M. Toth, Solid-state singlephoton emitters, Nat. Photonics 10, 631 (2016).

[57] M. Atatüre, D. Englund, N. Vamivakas, S. Y. Lee, and J. Wrachtrup, Material platforms for spin-based photonic quantum technologies, Nat. Rev. Mater. 3, 38 (2018).

[58] L. Childress, R. Walsworth, and M. Lukin, Atom-like crystal defects: From quantum computers to biological sensors, Phys. Today 67(10), 38 (2014).

[59] M. K. Bhaskar, R. Riedinger, B. Machielse, D. S. Levonian, C. T. Nguyen, E. N. Knall, H. Park, D. Englund, M. Lončar, D. D. Sukachev, and M. D. Lukin, Experimental demonstration of memory-enhanced quantum communication, Nature 580, 60 (2019).

[60] P. Narang, C. J. Ciccarino, J. Flick, and D. Englund, Quantum materials with atomic precision: Artificial atoms in solids: $A b$ initio design, control, and integration of single photon emitters in artificial quantum materials, Adv. Funct. Mater. 29, 1904557 (2019).

[61] P. F. Loos, M. Boggio-Pasqua, A. Scemama, M. Caffarel, and D. Jacquemin, Reference energies for double excitations, J. Chem. Theory Comput. 15, 1939 (2019).

[62] N. Nikolay, N. Mendelson, E. Özelci, B. Sontheimer, F. Böhm, G. Kewes, M. Toth, I. Aharonovich, and O. Benson, Direct measurement of quantum efficiency of single-photon emitters in hexagonal boron nitride, Optica 6, 1084 (2019).

[63] J. Iles-Smith, D. P. S. McCutcheon, A. Nazir, and J. Mørk, Phonon scattering inhibits simultaneous near-unity efficiency and indistinguishability in semiconductor singlephoton sources, Nat. Photonics 11, 521 (2017).

[64] R. J. Young, R. M. Stevenson, P. Atkinson, K. Cooper, D. A. Ritchie, and A. J. Shields, Improved fidelity of triggered entangled photons from single quantum dots, New J. Phys. 8, 29 (2006).

[65] H. Wang, H. Hu, T.-H. Chung, J. Qin, X. Yang, J.-P. Li, R.-Z. Liu, H.-S. Zhong, Y.-M. He, X. Ding, Y.-H. Deng, Q. Dai, Y.-H. Huo, S. Höfling, C.-Y. Lu, and J.-W. Pan, On-Demand Semiconductor Source Of Entangled Photons which Simultaneously has High fidelity, Efficiency, and Indistinguishability, Phys. Rev. Lett. 122, 113602 (2019).

[66] R. Trivedi, K. A. Fischer, J. Vučković, and K. Müller, Generation of non-classical light using semiconductor quantum dots, Adv. Quantum Technol. 3, 1900007 (2020).

[67] L. J. Rogers, S. Armstrong, M. J. Sellars, and N. B. Manson, Infrared emission of the NV center in diamond: Zeeman and uniaxial stress studies, New J. Phys. 10, 103024 (2008).

[68] S. A. Momenzadeh, R. J. Stöhr, F. F. De Oliveira, A. Brunner, A. Denisenko, S. Yang, F. Reinhard, and J. Wrachtrup, Nanoengineered diamond waveguide as a robust bright platform for nanomagnetometry using shallow nitrogen vacancy centers, Nano Lett. 15, 165 (2015).

[69] A. Faraon, C. Santori, Z. Huang, V. M. Acosta, and R. G Beausoleil, Coupling of Nitrogen-Vacancy Centers to Photonic Crystal Cavities in Monocrystalline Diamond, Phys. Rev. Lett. 109, 033604 (2012).

[70] C. Chakraborty, N. R. Jungwirth, G. D. Fuchs, and A. N. Vamivakas, Electrical manipulation of the fine-structure splitting of $\mathrm{WSe}_{2}$ quantum emitters, Phys. Rev. B 99, 45308 (2019).

[71] J. L. Zhang, S. Sun, M. J. Burek, C. Dory, Y. K. Tzeng, K. A. Fischer, Y. Kelaita, K. G. Lagoudakis, M. Radulaski, Z. X. Shen, N. A. Melosh, S. Chu, M. Lončar, and J. Vučković, Strongly cavity-enhanced spontaneous emission from siliconvacancy centers in diamond, Nano Lett. 18, 1360 (2018).

[72] B. Machielse, S. Bogdanovic, S. Meesala, S. Gauthier, M. J. Burek, G. Joe, M. Chalupnik, Y. I. Sohn, J. Holzgrafe, R. E. Evans, C. Chia, H. Atikian, M. K. Bhaskar, D. D. Sukachev, L. Shao, S. Maity, M. D. Lukin, and M. Lončar, Quantum Interference of Electromechanically Stabilized Emitters in Nanophotonic Devices, Phys. Rev. X 9, 031022 (2019).

[73] H. B. Chen, P. Y. Lo, C. Gneiting, J. Bae, Y. N. Chen, and F. Nori, Quantifying the nonclassicality of pure dephasing, Nat. Commun. 10, 3794 (2019).

[74] P. Tighineanu, C. L. Dreeßen, C. Flindt, P. Lodahl, and A. S. Sørensen, Phonon Decoherence of Quantum Dots in Photonic Structures: Broadening of the Zero-Phonon Line and the Role of Dimensionality, Phys. Rev. Lett. 120, 257401 (2018). 